\title{
Effect of crystal defects on the electrical properties in epitaxial tin dioxide thin films
}

\author{
J. E. Dominguez, L. Fu, and X. Q. Pan ${ }^{\text {a) }}$ \\ Department of Materials Science and Engineering, The University of Michigan, Ann Arbor, Michigan 48109
}

(Received 29 August 2002; accepted 26 October 2002)

\begin{abstract}
Epitaxial (101) tin dioxide thin films with thickness ranging from 6 and $100 \mathrm{~nm}$ were deposited on the (10 $\overline{1} 2) \alpha-\mathrm{Al}_{2} \mathrm{O}_{3}$ substrate by femtosecond pulsed laser ablation. Due to the lattice and thermal expansion mismatch with the substrate, the $\mathrm{SnO}_{2}$ film shows interfacial misfit dislocations, antiphase boundaries (APBs), and partial dislocations. The APBs lie along the ( $\overline{101})$ planes with a displacement of $1 / 2[101]$. The densities of APBs and partial dislocations vary with film thickness, whereas the average spacing of misfit dislocations remains constant. Hall effect measurements showed that both electron concentration and mobility decrease with a reduction in the film thickness, which is ascribed to the scattering of electrons by crystal defects and interfaces and the effect of a native space charge region at the near-surface region of the films. The response of the films to reducing gases was found to depend on the electron concentration of the film and the relative fraction, with respect to film thickness, of material that is depleted of electrons. (C) 2002 American Institute of Physics. [DOI: 10.1063/1.1530745]
\end{abstract}

The measurable interaction between semiconductor oxide surfaces and the ambient has resulted in a great number of practical applications, especially as sensor materials. By monitoring a given variable such as resistance or chemical potential, it is possible to obtain an input, although transient, from ambient conditions. The reliability and reproducibility of these sensors is dependent on their signal drift over time. Oxide sensors are usually affected by changes in bulk stoichiometry over time due to prolonged exposures to different atmospheres at relatively high temperatures. ${ }^{1}$ For example, drift in tin dioxide gas sensors is known to occur due to grain-boundary diffusion of oxygen species to and from the bulk. ${ }^{2}$ Our recent work showed that the properties of $\mathrm{SnO}_{2}$ thin films strongly depend on the microstructure and chemistry of the film. ${ }^{3-5}$ The presence of grain boundaries in gas sensors also implies complex responses due to the electron trap states formed at the interfaces, which result in back-toback Schottky barriers. ${ }^{6}$ The height of these barriers can be modulated by the adsorption or desorption of oxygen or other gaseous species. ${ }^{7}$ Furthermore, the catalytic activity at grain boundaries is not well understood and dopants or adsorbed species located inside them can give rise to nonlinear current-voltage relationships. ${ }^{8}$ These deleterious effects observed in ceramic pellet sensors can be avoided by the fabrication of ultrathin single-crystal film sensors. The most important advantages of thin-film sensors over bulk ceramic sensors reside on their fast response, sensitivity to very dilute gas concentrations, good reliability and reproducibility, and relatively simple fabrication. Heteroepitaxial $\mathrm{SnO}_{2}$ thin films, for gas sensors applications, have been fabricated on different orientations of the $\alpha-\mathrm{Al}_{2} \mathrm{O}_{3}$ substrate. ${ }^{9-12}$ We have reported the fabrication and microstructure of single-crystal $\mathrm{SnO}_{2}$ films deposited by femtosecond pulsed laser ablation on the (10 $\overline{1} 2) \alpha-\mathrm{Al}_{2} \mathrm{O}_{3}$ substrate. ${ }^{12}$ In this letter, we report

${ }^{a)}$ Electronic mail: panx@umich.edu our studies on the structure-property relationships of singlecrystal $\mathrm{SnO}_{2}$ films with different thicknesses.

Tin oxide films were deposited on a (1012) $\alpha-\mathrm{Al}_{2} \mathrm{O}_{3}$ substrate using femtosecond pulsed laser ablation. The deposition conditions used were the same as reported previously, with a laser wavelength of $780 \mathrm{~nm}$ and a pulse width of 100 fs. ${ }^{12}$ The substrates were resistively heated to $700{ }^{\circ} \mathrm{C}$ and a background oxygen pressure of 0.1 mTorr was used during deposition. The microstructure of the films was studied using transmission electron microscopy (TEM) and x-ray diffraction. All films were deposited on the substrates cut from the same 3 in. wafer. X-ray diffraction analyses $(\theta-2 \theta$ and pole figures, not shown here) indicate that all the films were grown epitaxially on the substrate with $\mathrm{SnO}_{2}(101)$ $\times[010] / / \mathrm{Al}_{2} \mathrm{O}_{3}(10 \overline{1} 2)[1 \overline{2} 10]$. The full width at half maximum from rocking curves also indicated high crystallinity, with values ranging from $0.5^{\circ}$ for the $15 \mathrm{~nm}$ thick film to $1.2^{\circ}$ for the $100 \mathrm{~nm}$ thick ones.

The lattice mismatch between the (101) $\mathrm{SnO}_{2}$ film and the (1012) sapphire substrate is $11.42 \%$ along the [010] direction and $-0.4 \%$ along the $[\overline{101}]$ direction of $\mathrm{SnO}_{2}$. Misfit dislocations with Burger's vector $\mathbf{b}=1 / 2[\overline{101}]$ were observed along the [010] direction of $\mathrm{SnO}_{2}$, but no dislocations or planar defects are observed when the film is viewed in the [101] direction (see Fig. 3 in Ref. 12). Figures 1(a) and 1(b) are dark-field images of $15 \mathrm{~nm}$ and $60 \mathrm{~nm}$ thick films, both taken with electron beam incident along the $\mathrm{SnO}_{2}[010]$ direction. The parallel fringes in these images are coherent crystallographic shear planes (CSPs), which are usually called antiphase boundaries (APBs). The APBs lie on the (101) planes with a displacement vector of $1 / 2[101]$. By comparing Figs. 1(a) with 1(b), it can be seen that the spacing of APBs decreases with film thickness and that APBs tend to terminate inside the film as the film thickness increases. The termination of an APB in the film will create a partial dislocation with the Burger's vector 1/2[101]. Figure 
(a)

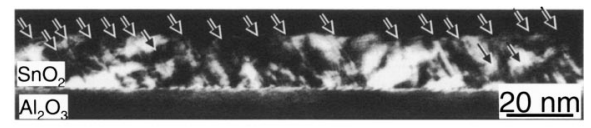

(b)

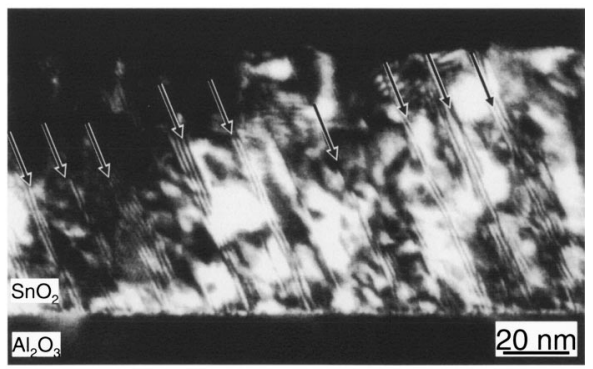

(c)

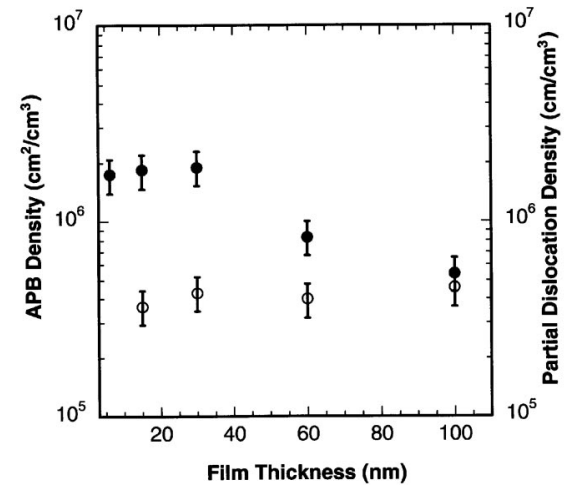

FIG. 1. (a) Dark-field image of the $15 \mathrm{~nm}$ and (b) $60 \mathrm{~nm}$ thick $\mathrm{SnO}_{2}$ film viewed in the [010] direction showing planar defects (arrows). (c) The densities of APBs and partial dislocations as a function of film thickness.

1(c) shows the total density of APBs and the partial dislocations as function of the film thickness. One sees that the density of APBs decreases with increasing film thickness. On the other hand, the density of partial dislocations increases with film thickness.

The occurrence of CSPs in the rutile structure could be caused by thermal stresses or defect clustering due to nonstoichiometry. ${ }^{13,14}$ In the present situation, the shear direction is in the shear plane, which indicates that the stoichiometry is conserved everywhere except at the partial dislocation at the end of the APBs. ${ }^{15}$ In addition, the distance between coherent CSPs is random in epitaxial $\mathrm{SnO}_{2}$ films, in contrast to the ordered CSP arrays found in nonstoichiometric titanium oxides. ${ }^{16}$ Furthermore, it is well known that in the rutile structure the most favorable slip planes correspond to the $\{101\}$ family of planes,${ }^{17}$ which is consistent with our observations [Figs. 1(a) and 1(b)]. On the other hand, x-ray diffraction and cross-sectional TEM studies showed that the $\mathrm{SnO}_{2}$ film is misoriented from the substrate about $1^{\circ}$. Thus, it is possible that thermal stresses or substrate steps are the contributing factor to the formation of coherent CPSs.

Figure 2 is a high-resolution TEM image of the interface between a $6 \mathrm{~nm}$ thick $\mathrm{SnO}_{2}$ film and the sapphire substrate. Quasiperiodic misfit dislocations at the interface are clearly visible, as well as some APBs. The missing half planes are located in $\mathrm{SnO}_{2}$ because the film is compressively strained. The Burger's vector of the dislocations is $1 / 2[\overline{101}]$ in the $\mathrm{SnO}_{2}$ film. The spacing between dislocations for a completely strain-relaxed film is calculated to be $2.48 \mathrm{~nm}$. The spacing is nearly constant $(\sim 2.5 \mathrm{~nm})$ for all films studied,

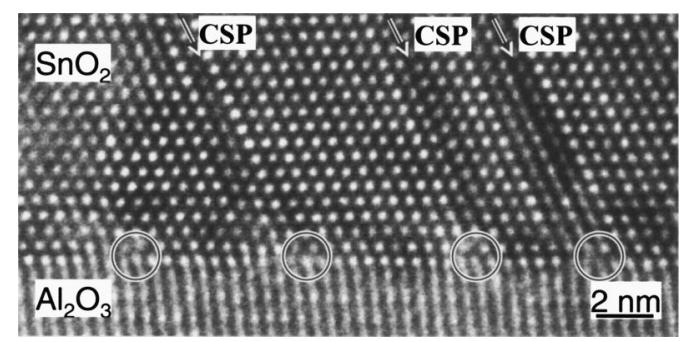

FIG. 2. (a) High-resolution TEM image of the (101) $\mathrm{SnO}_{2} /(10 \overline{1} 2) \mathrm{Al}_{2} \mathrm{O}_{3}$ interface viewed in the $\mathrm{SnO}_{2}[010]$ direction showing misfit dislocations (circled) and APBs (arrows). (b) Average spacing between misfit dislocations a function of film thickness.

which corresponds to a relaxation of about $90 \%$ of the interfacial mismatch strain.

Four-point probe resistance and Hall effect measurements were conducted. Figure 3(a) shows the electrical conductivity as function of inverse absolute temperature, for the epitaxial films with three different thicknesses, 6, 60, and $100 \mathrm{~nm}$. A difference in conductivity of about 4 orders of magnitude is observed between the $6 \mathrm{~nm}$ and $100 \mathrm{~nm}$ thick films. Figures 3(b) and 3(c) show the calculated carrier density and mobility of the films as a function of inverse temperature. The carrier density and mobility decrease with reducing film thickness. The activation energy, calculated from the slope of Fig. 3(b) $\left(N=K \exp \left(-E_{a} / k T\right)\right.$, where $E_{a}$ is the activation energy) are $0.52 \mathrm{eV}, 0.11 \mathrm{eV}$, and $0.07 \mathrm{eV}$ for the 6,60 , and $100 \mathrm{~nm}$ thick films, respectively. The difference in transport properties of the films is caused by the interaction between electrons and crystal defects in thin films and also from the effect of the film surface and the film/substrate interface. Since all films studied in this work were deposited under the same conditions except for different film thickness, we neglect the effect of point defects (mainly oxygen vacancies) in the present work. Crystal defects such as dislocations and APBs can act as scattering centers for electrons ${ }^{18}$ due to the distortion of the crystal lattice near the defects, which decreases the mobility of the electrons. Studies of planar defects in $\mathrm{CdS}^{19}$ have shown that the mobility can be signifi-

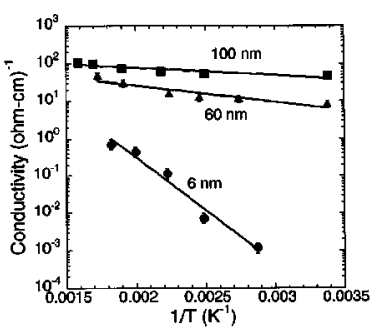

(a)

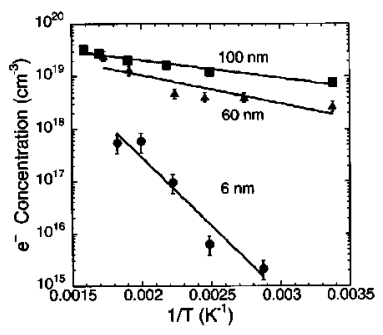

(b)

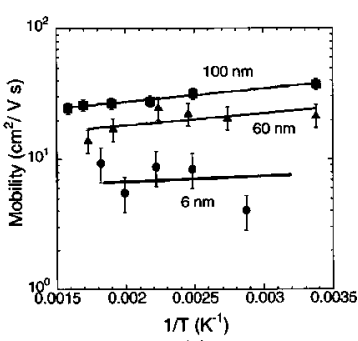

(c)

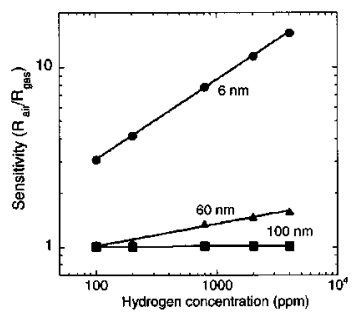

(d)
FIG. 3. Electrical transport properties of films with different thicknesses as a function of inverse absolute temperature; (a) conductivity, (b) electron concentration, (c) mobility, and (d) response of the same films to hydrogen. 
cantly reduced for films with planar defect densities greater than $10^{3}$ faults $/ \mathrm{cm}$, much lower than the density found in the $\mathrm{SnO}_{2}$ films shown here. For a highly faulted material, the mobility $\mu$ has been considered similar to materials with grain boundaries:

$$
\mu \propto \mu_{b}\left[1+m \exp \left(\frac{q \phi_{f}}{k T}\right)\right]^{-1},
$$

where $m$ is the defect density, $q$ is the electron charge, $\phi_{f}$ is the potential at the stacking fault, and $\mu_{b}$ is the bulk mobility. Equation (1) predicts a decrease in mobility with increasing defect density and temperature, as seen in Fig. 3(c). On the other hand, misfit dislocations also create a potential well at their cores and electrical barriers beside the well, which reduce the electron mobility. ${ }^{20}$ This effect becomes more pronounced for thinner films. Also, the film surface and the interface between the film and substrate can scatter conduction electrons. As the film thickness gets closer to the electron mean-free path in bulk $\mathrm{SnO}_{2}$ (about $8 \mathrm{~nm}$ using 250 $\mathrm{cm}^{2} / \mathrm{V}$ s for bulk $\mathrm{SnO}_{2}$ mobility), the scattering effects of interfacial defects, film surface, and film/substrate interface increase, reducing the electron mobility in the film.

CSPs can also influence the free electron concentration of the film. Previous studies ${ }^{15,21}$ have suggested that coherent CSPs terminating inside $\mathrm{SnO}_{2}$ film may produce oxygen vacancies. Coherent CSPs that terminate at the film surface do not change the film stoichiometry and thus have no effect on the electron concentration of the film. Since the free electrons in pure $\mathrm{SnO}_{2}$ come primarily from oxygen vacancies, a greater number of APBs terminating inside the film creates higher electron concentration. Thus, as the film thickness increases, the electron concentration of the film also increases due to the increased number of APBs that terminate inside the film, as shown in Fig. 1(c). Furthermore, charged APBs can create traps for free electrons that further reduce the electron density in the films.

Another factor decreasing electron concentration and mobility with film thickness can be due to the native space charge of the films. A native space charge appears due to the presence of surface states. These surface states can appear due to ionized oxygen adsorbed during high-temperature deposition and subsequent cooling or negatively charged native ionic species at the surface, such as surface lattice $\mathrm{O}^{-}$, which is known to appear at high temperatures on oxide surfaces. ${ }^{22}$ The presence of a negative charge at the surface will result in an opposite charge at the near surface or a depletion region for an $n$-type semiconductor. Thus, the effect of having a native negative charge at the surface will result in a decrease in electron concentration in the nearsurface region of the film. Since the charge trapped at the surface is a two-dimensional charge density, the volume density of free electrons in the film will decrease with decreasing thickness. The concentration of charge at the near surface can also act as a scattering center due to the band bending and energy difference between bulk and surface electrons. Thus, the mobility will also be reduced with decreasing film thickness as shown in Fig. 3(c).

To test the response of the films to reducing gases, the films were heated to $365^{\circ} \mathrm{C}$ in a reactor with flowing synthetic air $\left(80 \% \mathrm{~N}_{2}\right.$ and $\left.20 \% \mathrm{O}_{2}\right)$ and hydrogen introduced at concentrations from 100 to $4000 \mathrm{ppm}$. Figure 3(d) shows the sensitivity to hydrogen of the same films as in Fig. 3(a)-3(c). The sensitivity ${ }^{23}$ to hydrogen increases with reducing film thickness with a clear maximum in sensitivity for the $6 \mathrm{~nm}$ thick film. This is a consequence of two synergetic factors: Film thickness and electron concentration. Oxygen adsorption on an $n$-type semiconductor traps free electrons at the surface creating a negative charge at the surface that is compensated by an electron depletion zone near the surface. This depletion layer (Debye length) depends on electron concentration. The depletion width at about $350{ }^{\circ} \mathrm{C}$ would be about $5 \mathrm{~nm}, 2 \mathrm{~nm}$, and $1 \mathrm{~nm}$ for the films $6 \mathrm{~nm}, 60 \mathrm{~nm}$, and $100 \mathrm{~nm}$ thick, respectively. The introduction of a reducing gas creates a dynamic equilibrium wherein the oxygen coverage is reduced and the electron concentration is increased with a consequent increase in the film conductivity. On the other hand, as the films become thinner, the depletion length is of the same magnitude as the film thickness and the relative change in conductivity with the introduction of a reducing gas is greater. The sensitivity of the film increases with decreasing film thickness.

In conclusion, the microstructure and electrical properties of heteroepitaxial $\mathrm{SnO}_{2}$ films with varying thickness were analyzed. It was found that crystal defects (misfit dislocations and APBs) in the films have strong effects on the electron concentration and mobility of the films. The sensitivity to reducing gases was found to increase with reducing film thickness due to a greater extent of electron depletion as the film thickness decreased.

This work was supported by the National Science Foundation (NSF) through Grant Nos. NSF/DMR 9875405 (CAREER, XQP) and DMR/IMR 9704175.

${ }^{1}$ T. A. Jones, in Solid State Gas Sensors, edited by P. T. Moseley, B. C. Tofield, and A. Hilger (IOP, Bristol, 1987), pp. 51-70.

${ }^{2}$ W. Gopel and K. D. Schierbaum, Sens. Actuators B 26, 1 (1995).

${ }^{3}$ X. Q. Pan and L. Fu, J. Electroceram. 7, 35 (2001).

${ }^{4}$ X. Q. Pan and L. Fu, J. Appl. Phys. 89, 6048 (2001).

${ }^{5}$ X. Q. Pan, L. Fu, and J. E. Dominguez, J. Appl. Phys. 89, 6056 (2001).

${ }^{6}$ F. Greuter and G. Blatter, Semicond. Sci. Technol. 5, 111 (1990).

${ }^{7}$ K. Ihokura, New Mater. Proc. 1, 43 (1981).

${ }^{8}$ P. R. Bueno, S. A. Pianaro, E. C. Pereira, L. O. S. Bulhoes, E. Longo, and J. A. Varela, J. Appl. Phys. 84, 3700 (1998).

${ }^{9}$ S. Semancik and R. E. Cavicchi, Thin Solid Films 206, 81 (1991).

${ }^{10}$ J. E. Dominguez, L. Fu, and X. Q. Pan, Appl. Phys. Lett. 79, 614 (2001).

${ }^{11}$ D. S. Lee, G. H. Rue, J. S. Huh, S. D. Choi, and D. D. Lee, Sens. Actuators B 77, 90 (2001).

${ }^{12}$ J. Dominguez, L. Fu, and X. Pan, J. Appl. Phys. 91, 1060 (2002).

${ }^{13}$ C. R. A. Catlow, in Nonstoichiometric Oxides, edited by O. T. Sorensen (Academic, New York, 1981), pp. 61-98.

${ }^{14}$ M. G. Blanchin and G. Fontaine, Phys. Status Solidi A 29, 491 (1975).

${ }^{15}$ J. G. Zheng, X. Pan, M. Schweizer, U. Weimar, W. Gopel, and M. Ruhle, J. Mater. Sci. 31, 2317 (1996).

${ }^{16}$ A. M. Stoneham and P. J. Durham, J. Phys. Chem. Solids 34, 2127 (1973).

${ }^{17}$ K. G. Ashbee and R. E. Smallman, J. Am. Ceram. Soc. 46, 211 (1963).

${ }^{18}$ H. F. Mataré, Defect Electronic in Semiconductors (Wiley, New York, 1971), pp. 57-69.

${ }^{19}$ L. L. Kazmerski, W. B. Berry, and C. W. Allen, J. Appl. Phys. 43, 3521 (1972).

${ }^{20}$ R. Jaszek, J. Mater. Sci. 12, 1 (2001).

${ }^{21}$ J. G. Zheng, X. Pan, M. Schweizer, U. Weimar, W. Gopel, and M. Ruhle, Philos. Mag. Lett. 73, 93 (1996).

${ }^{22}$ M. J. Madou and S. R. Morrison, Chemical Sensing with Solid State Devices (Academic, San Diego, 1989), pp. 163-166.

${ }^{23}$ Sensitivity is defined as the ratio between the resistance of the film in air to that in a reducing atmosphere. 